\title{
Nidificação de Centris (Hemisiella) tarsata Smith (Hymenoptera, Apidae, Centridini) em ninhos-armadilha no Nordeste do Maranhão, Brasil ${ }^{1}$
}

\author{
Fernanda N. Mendes² \& Márcia M. C. Rêgo ${ }^{3}$
}

\begin{abstract}
${ }^{1}$ Monografia apresentada ao curso de Ciências Biológicas da Universidade Federal do Maranhão, São Luís-MA, Brasil. ${ }^{2}$ Bolsista do programa PIBIC / CNPq / UFMA. fnmodel@yahoo.com.br ${ }^{3}$ Departamento de Biologia, Universidade Federal do Maranhão. Av. dos Portugueses, s/n, Bacanga, 65080-581 São Luís-MA, Brasil. regommc@uol.com.br
\end{abstract}

\begin{abstract}
Nidification of Centris (Hemisiella) tarsata Smith (Hymenoptera, Apidae, Centridini) in trap nests in Northeast Maranhão, Brazil. This work had as objective to obtain ecological data of Centris (Hemisiella) tarsata Smith's nidification in three ecosystems: riparian forest (MC), mesophitic forest (MM) and eucalyptal (EC), using trap nests made by bamboo canes, distributed in differentiated heights: $1,5 \mathrm{~m}$ and 5-12 m high. A total of 41 nests were collected: 31 in $\mathrm{EC}$ and 10 in $\mathrm{MM}$, the majority in the upper strata and with the largest frequency of nesting occurring in the dry season. The sex ratio was of 1.9:1 (females/ males) in EC and of 1.08:1 in MM. About $22 \%$ of nests of the EC and $40 \%$ of MM were parasitized by Mesocheira bicolor (Fabricius 1804) (Hymenoptera, Apidae) and Coelioxys sp. (Hymenoptera, Megachilidae). The pollinic analyses showed a higher quantity of pollen grains of Banisteriopsis sp. (Malpighiaceae) and Cassia sp. (Caesalpiniaceae) in EC area and a species of Caesalpiniaceae Kunth. and Banisteriopsis Robinson in MM area.
\end{abstract}

KEYWORDS. Bamboo; bees; pollen.

RESUMO. Nidificação de Centris (Hemisiella) tarsata Smith (Hymenoptera, Apidae, Centridini) em ninhos-armadilha no Nordeste do Maranhão, Brasil. Este trabalho teve como objetivo obter dados sobre a ecologia da nidificação de Centris (Hemisiella) tarsata Smith em três ecossistemas: mata ciliar (MC), mata mesofítica (MM) e eucaliptal (EC), utilizandose ninhos-armadilha confeccionados em gomos de bambu, distribuídos em diferentes alturas: 1,5 m e 5-12 m do solo. Foram obtidos 41 ninhos: 31 no EC e 10 na MM, a maioria no estrato superior e com maior frequiência de nidificações ocorrendo no período de estiagem. A razão sexual foi de 1,9:1 (fêmeas/ machos) no EC e de 1,08:1 na MM. Cerca de 22\% dos ninhos do EC e 40\% da MM foram parasitados por Mesocheira bicolor (Fabricius, 1804) (Hymenoptera, Apidae) e Coelioxys sp. (Hymenoptera, Megachilidae). A análise polínica revelou predominância de grãos de pólen de Banisteriopsis sp. (Malpighiaceae) e Cassia sp. (Caesalpiniaceae) no EC e de espécies de Caesalpiniaceae Kunth. e Banisteriopsis Robinson na MM.

PALAVRAS-CHAVE. Abelhas; bambu; pólen.

As abelhas da tribo Centridini Cockerell \& Cockerell são solitárias e possuem diversos hábitos de nidificação, dentre estes o de utilizar cavidades preexistentes. Espécies como Centris segregata Crawford, 1906, Centris trigonoides Lepeletier, 1841, Centris vittata Lepeletier, 1941, Centris analis (Fabricius, 1804) e Centris tarsata Smith, 1874, nidificam nessas cavidades e, por isso, podem estabelecer seus ninhos em ninhos-armadilha (Coville et al. 1983; Pereira et al. 1999; Jesus \& Garófalo 2000; Silva et al. 2001; Aguiar \& Garófalo 2004).

Segundo Vinson et al. (1993) estas cavidades representam um recurso escasso no ambiente, o que limita o crescimento das populações de abelhas e vespas que as utilizam. Diante disso, a ausência ou um acentuado declínio de polinizadores em determinadas localidades do mundo tem estimulado o uso de ninhos-armadilha para proporcionar locais de nidificação e, conseqüentemente, aumento das populações e assim garantir a polinização de diversas culturas (Roubik 1995; Sampson \& Cane 2000; Bosh \& Kemp 2002; Garófalo et al. 2004). Além disso, as espécies que nidificam em ninhosarmadilha também são utilizadas como bioindicadores de qualidade do ambiente e de diversidade em programas de conservação ambiental (Beyer et al. 1987; Frankie et al. 1998; Tscharntke et al. 1998; Morato \& Campos 2000).
As abelhas Centridini são conhecidas por serem especializadas na coleta de óleos florais, os quais são utilizados na construção de partes do ninho e para a alimentação das larvas (Buchmann 1987; Vogel 1974). Machado (2004) citou seis famílias de plantas visitadas por espécies de Centris Fabricius, 1804 para coleta de óleo: Iridaceae Juss., Orchidaceae Juss., Malpighiaceae Juss., Krameriaceae Dum., Scrophulariaceae Juss. e Solanaceae Juss. Dentre as Malpighiaceae, as plantas do gênero Byrsonima Rich. são freqüentemente visitadas por Centris (Gottsberger 1986; Albuquerque \& Rêgo 1989; Rêgo \& Albuquerque 1989, 2006; Sazima \& Sazima 1989; Barros 1992; Vinson et al. 1997; Teixeira $\&$ Machado 2000; Gaglianone 2003).

Diversos trabalhos com ninhos-armadilha já foram realizados no Brasil em áreas de Mata Atlântica (Aguiar \& Martins 2002), Floresta de Araucárias (Buschini 2006; Buschini $\&$ Wolff 2006), fragmentos de mata (Loyola \& Martins 2006), dunas litorâneas (Silva et al. 2001; Viana et al. 2001), caatinga e floresta estacional semi-decídua (Aguiar \& Garófalo 2004; Aguiar et al. 2005), entre outros. No Maranhão, entretanto, existe uma escassez de trabalhos sobre comunidades de abelhas solitárias que nidificam em cavidades preexistentes.

Este trabalho tem como objetivo obter dados sobre a 
ecologia da nidificação de Centris (Hemisiella) tarsata em três diferentes ecossistemas (mata ciliar, mata mesofítica e eucaliptal) inseridos no bioma cerrado do Maranhão.

\section{MATERIALE MÉTODOS}

As áreas de estudo estão localizadas em três fazendas de propriedade da Comercial e Agrícola Paineiras, no município de Urbano Santos, nordeste do Maranhão ( $3^{\circ} 12^{\prime} 28^{\prime \prime}$ S; $43^{\circ} 24^{\prime} 12^{\prime \prime} \mathrm{W}$ ).

O clima da região é tropical megatérmico (tipo Aw', na classificação de Köppen), com totais pluviométricos anuais em torno de $1800 \mathrm{~mm}$, sendo $89 \%$ desse total concentrado nos meses de dezembro a maio, com os meses de julho a outubro muito secos; a temperatura média anual varia entre $26^{\circ} \mathrm{C}$ e $27^{\circ} \mathrm{C}$. A vegetação predominante na região é o Cerrado, ocorrendo ainda formações de Floresta Estacional Semidecídua e manchas de vegetação mista (Brasil 1984).

As áreas de amostragem foram 4 ha de mata ciliar (MC), 1 ha isolado de mata mesofítica (MM) circundado por monoculturas de eucalipto e 1 ha de eucaliptal (EC) circundado por cerrado. A distância entre as áreas são: mata ciliar - mata mesofítica: 9,5 km; mata ciliar - eucaliptal: 9,7 km; mata mesofítica-eucaliptal: $17,9 \mathrm{~km}$.

Para a confecção dos ninhos-armadilha foram utilizados gomos de bambu fechados em uma das extremidades pelo próprio nó e cortados longitudinalmente, sendo as duas partes unidas com fita adesiva. Estes foram amarrados em conjuntos de cinco unidades com diferentes diâmetros e comprimentos, os quais variavam de $0,7 \mathrm{~cm}$ a $1,9 \mathrm{~cm}$ e de $11 \mathrm{~cm}$ a $24 \mathrm{~cm}$, respectivamente.

Em cada área foram colocados 40 conjuntos de bambus: 20 conjuntos a aproximadamente $1,5 \mathrm{~m}$ do solo, sendo presos às árvores com tiras de borracha, e 20 próximos ao dossel ( 5 a 12 $\mathrm{m}$ de altura), pendurados por uma corda de nylon que funcionava como roldana.

No EC os ninhos foram dispostos ao longo de quatro fileiras, nas quais foram selecionadas cinco árvores, cada uma com um conjunto no estrato inferior e outro no superior (dossel). Nas MM e MC os ninhos foram colocados ao longo de uma trilha preexistente no interior de cada área. Dessa maneira, cada área amostrada possuía 200 bambus, totalizando 600 ninhos-armadilha. Os ninhos-armadilha foram vistoriados mensalmente, com auxílio de um otoscópio, de agosto de 2002 a julho de 2003.

Os ninhos fundados foram levados para o laboratório e colocados em caixas de isopor ( $13,0 \times 10,5 \times 5,5 \mathrm{~cm})$, cobertas com vidro. Os indivíduos que emergiram foram sacrificados em câmaras com acetato de etila, alfinetados e etiquetados. Após a emergência dos indivíduos, os ninhos foram abertos para a conferência e medição de células (comprimento, diâmetro, disposição). O tamanho dos machos e das fêmeas foi determinado pela medida da largura máxima da cabeça. As abelhas foram depositadas na coleção entomológica do Laboratório de Estudos sobre abelhas (LEA) da Universidade Federal do Maranhão (UFMA).
Os grãos de pólen encontrados nas células dos ninhos (com e sem oviposição) foram coletados após a emergência de todos os indivíduos e colocados em vidros com etiquetas identificando o ninho de origem. Este material foi acondicionado na geladeira para posterior confecção das lâminas polínicas, de acordo com o Método da Acetólise descrito por Erdtman (1966). Foram preparadas três lâminas de cada amostra $(\mathrm{n}=$ 27), das quais duas foram enviadas para análise no Museu Paraense Emílio Goeldi (Belém-PA).

Os dados de temperatura e umidade foram tomados nas áreas de estudo com auxílio de termohigrômetro e a pluviosidade mensal da cidade foi fornecida pela Comercial e Agrícola Paineiras, em Urbano Santos (MA).

$\mathrm{O}$ teste $\mathrm{t}$ foi utilizado para verificar a ocorrência de diferenças significativas entre os tamanhos médios (largura máxima da cabeça) de machos e fêmeas e suas respectivas células. $\mathrm{O}$ teste do qui-quadrado $\left(x^{2}\right)$ foi empregado para analisar se houve diferenças significativas entre as proporções esperadas e obtidas de machos e fêmeas, e a correlação de Spearman (r) para observar possíveis correlações entre o número de ninhos e a pluviosidade, bem como entre o tamanho dos indivíduos e o comprimento e diâmetro das células.

\section{RESULTADOS}

Foram coletados 41 ninhos de $C$. tarsata: 31 no eucaliptal, representando $15,5 \%$ do total de ninhos colocados nesta área, e $10(5 \%)$ na mata mesofítica; na mata ciliar não ocorreu nidificação.

As nidificações ocorreram no período de estiagem, de agosto a dezembro de 2002, sendo que as maiores freqüências ocorreram em setembro e outubro no EC, e em agosto na MM, havendo queda no número de ninhos fundados nos meses posteriores. Observou-se uma significativa correlação negativa $(\mathrm{r}=-0,43 ; \mathrm{p}<0,05)$ entre o número de ninhos coletados e a precipitação pluviométrica (Fig. 1).

Quanto à altura dos ninhos, a maioria dos bambus utilizados estava no estrato superior (5 a $12 \mathrm{~m}$ ): 18 (58\%) no EC e 6 (60\%) na MM.

Não houve diferença no comprimento $(t=-0,18 ; \mathrm{p}<0,05) \mathrm{e}$ no diâmetro $(\mathrm{t}=0,52 ; \mathrm{p}<0,05)$ dos bambus utilizados no EC e na MM. O comprimento dos bambus nas duas áreas variou de 11,5 a $24 \mathrm{~cm}(\bar{x}=20,5 \mathrm{~cm} \pm 2,3 ; \mathrm{n}=41)$, e o diâmetro de 0,7 a $1,9 \mathrm{~cm}(\bar{x}=1,0 \mathrm{~cm} \pm 0,24 ; \mathrm{n}=41)$ (Fig. 2).

Houve baixas correlações entre as medidas dos bambus e o número e as medidas das células no ninho (Tab. I), sendo observadas de 1 a 12 células ( $\bar{x}=4,2 \pm 2,79 ; \mathrm{n}=132$ ) por ninho no EC e de 2 a $8(\bar{x}=5,7 \pm 2,11 ; \mathrm{n}=57)$ na MM. Algumas destas células se encontravam somente com pólen $(\mathrm{n}=8)$, vazias $(\mathrm{n}=$ 6) ou incompletas $(n=3)$. O material utilizado na construção das células constituía-se de areia na parte externa e uma substância semelhante a resina ou óleo na parte interna, tendo esta aspecto polido e brilhante. As células se encontravam em sequiência linear, oblíquas em relação ao plano horizontal, na maior parte dos ninhos $(\mathrm{EC}-\mathrm{n}=19$; $\mathrm{MM}-\mathrm{n}=6$ ), ou menos frequientemente dispostas uma ao lado da outra, em duas fileiras paralelas $(E C-n=4)$. 


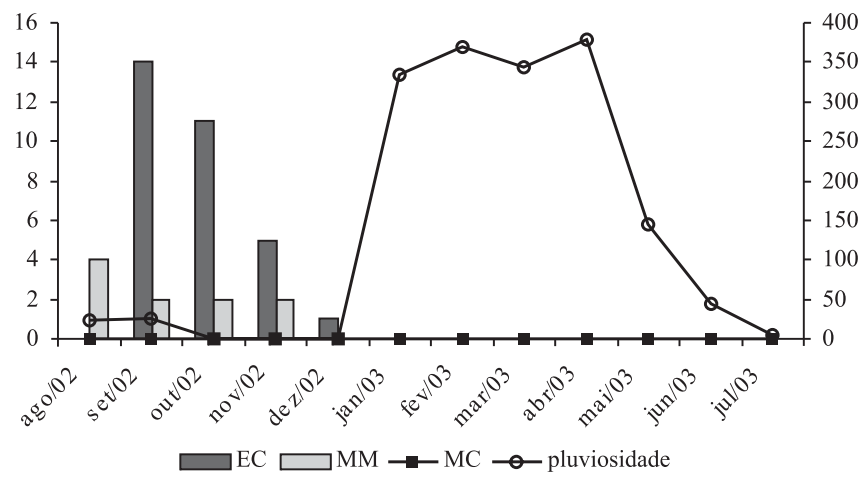

Fig. 1. Variação nos índices mensais de pluviosidade e freqüência de ninhos de Centris tarsata coletados de agosto/ 2002 a julho/ 2003 em eucaliptal (EC), mata mesofítica (MM) e mata ciliar (MC), Urbano Santos, MA, Brasil.

Tanto no EC como na MM houve protandria, emergindo 29 fêmeas e 15 machos dos ninhos do EC, representando uma razão sexual de 1,9:1, diferindo significativamente de $1: 1\left(x^{2}=\right.$ 6,24; $\mathrm{gl}=1 ; \mathrm{p}<0,007)$, e 13 fêmeas e 12 machos $(1,08: 1)$ da MM, não havendo diferença significativa de $1: 1\left(x^{2}=0,00 ; \mathrm{gl}\right.$ $=1 ; \mathrm{p}<0,841)$.

O intervalo entre a coleta dos ninhos e a emergência dos indivíduos variou de 2 a 7 semanas, aproximadamente. Nas duas áreas foi observada apenas uma geração por ano, com um curto ciclo de vida, sendo no EC de setembro/ outubro a novembro/ dezembro, e na MM de agosto/ setembro a outubro/ novembro (Fig. 3). C. tarsata apresentou desenvolvimento direto, sem diapausa na fase de larva.

As fêmeas apresentaram largura da cabeça maior que os machos nas duas áreas $(\mathrm{t}=7,1 ; \mathrm{p}<0,05)$. A largura da cabeça das fêmeas e dos machos variou de 3,6 a 4,7 $\mathrm{mm}(\bar{x}=4,3 \pm 0,21$; $\mathrm{n}=41)$ e 3,3 a $4,8 \mathrm{~mm}(\bar{x}=3,8 \pm 0,26 ; \mathrm{n}=26)$, respectivamente.

As células das fêmeas apresentaram comprimento de 1,0 a $1,4 \mathrm{~cm}(\bar{x}=1,1 \pm 0,1)$ e diâmetro de 0,6 e $0,7 \mathrm{~cm}(\bar{x}=0,6 \pm 0,05$; $\mathrm{n}=41$ ), sendo maiores que as dos machos, com comprimento de 0,8 a $1,3 \mathrm{~cm}(\bar{x}=1,0 \pm 0,1)$ e diâmetro de 0,5 a $0,7 \mathrm{~cm}(\bar{x}=0,6$ $\pm 0,05 ; \mathrm{n}=26)$, mas a diferença não foi significativa (comprimento $-\mathrm{t}=0,82 ; \mathrm{p}<0,05$; diâmetro $-\mathrm{t}=1,59 ; \mathrm{p}<0,05)$. Entretanto, houve correlação positiva significativa, ainda que baixa $(\mathrm{r}=0,30 ; \mathrm{p}<0,05)$, entre o diâmetro das células e a largura da cabeça de machos e fêmeas. Não houve correlações significativas entre as medidas dos bambus e das células (Tab. I).

No EC, 22,5\% dos ninhos foram parasitados, sendo cinco por Mesocheira bicolor (Fabricius, 1804) (Hymenoptera, Apidae) e dois por Coelioxys (Acrocoelioxys) sp. (Hymenoptera, Megachilidae), emergindo seis e cinco indivíduos, respectivamente. Na MM, 40\% dos ninhos foram parasitados, sendo oito por Coelioxys (Acrocoelioxys) sp., emergindo oito indivíduos, e um por $M$. bicolor, emergindo apenas um indivíduo.

Nos ninhos do EC foram identificados grãos de pólen de oito gêneros vegetais e de duas espécies não identificadas
(Caesalpiniaceae Kunth. e Myrtaceae Juss.), sendo observada maior quantidade de grãos de Banisteriopsis Robinson (Malpighiaceae - 22 lâminas: 64,7\%) e Cassia L. (Caesalpiniaceae - 17 lâminas: 50\%) (Tab. II). Na MM foram diagnosticados grãos de pólen de cinco gêneros vegetais e de uma Caesalpiniaceae não identificada, sendo observado maior número de grãos de pólen da Caesalpiniaceae (6 lâminas: 54,5\%) e de Banisteriopsis (4 lâminas: 36,3\%) (Tab. II).

\section{DISCUSSÃO}

Comparando-se as áreas amostradas, Centris tarsata nidificou mais no eucaliptal $(75,6 \% ; n=31)$. No nordeste do Brasil, esta espécie nidifica com frequiência em hábitats quentes e ensolarados com vegetações abertas (Aguiar \& Garófalo 2004), como em ambiente de dunas (Viana et al. 2001; Silva et al. 2001) e caatinga (Aguiar \& Martins 2002), mas também ocorre em menor intensidade em fragmentos de floresta no sudeste do país (Camillo et al. 1995). O EC, por ser uma área predominantemente aberta, recebe incidência solar uniforme em toda a sua extensão, o que torna sua temperatura e umidade favoráveis à nidificação dessa espécie.

$\mathrm{O}$ isolamento de uma porção de mata (como o fragmento de mata mesofítica estudado) é seguido por mudanças físicas que são responsáveis por muitas modificações observadas nas comunidades e na biologia de animais que nela existem (Lovejoy et al. 1986). Essas modificações podem desfavorecer espécies adaptadas ao interior da mata e beneficiar aquelas adaptadas a ambientes abertos, como $C$. tarsata, que nidificou em pequeno número na MM. Essa baixa freqüência pode ter ocorrido em virtude da diversidade de substratos naturais aí existentes e da umidade elevada no interior da área, o que proporcionou condições para proliferação de fungos, cupins e formigas nos bambus.

A ausência de ninhos de $C$. tarsata na MC pode ter sido influenciada pela presença de ninhos-armadilha confeccionados em madeira, estudados por Moreira et al. (2002). Estes autores coletaram 119 ninhos de Centris tarsata neste tipo de substrato durante dois anos de coleta (set/ 2001 a out/ 2003). Comparando os dados desses autores com os obtidos aqui, pode-se sugerir uma possível preferência de $C$. tarsata por substrato de madeira, uma vez que antes da colocação destes na área de estudo foram obtidos em bambu 2 ninhos de $C$. tarsata e 3 de $C$. (Hemisiella) sp. (Mendes \& Rêgo 2002). A preferência por madeira talvez ocorra devido à semelhança desse substrato aos locais naturais de nidificação.

Tabela I. Correlação entre o número e o tamanho das células de Centris tarsata e dos bambus nidificados $(\mathrm{n}=67$ células; $\mathrm{p}<0,05)$ em Urbano Santos, MA, Brasil.

\begin{tabular}{lccc}
\hline & $\begin{array}{c}\mathrm{N}^{\mathbf{0}} \\
\text { de células }\end{array}$ & $\begin{array}{c}\text { Comprimento } \\
\text { das células }\end{array}$ & $\begin{array}{c}\text { Diâmetro } \\
\text { das células }\end{array}$ \\
\hline Compr. do bambu & 0,17 & $-0,04$ & 0,04 \\
Diâm. do bambu & 0,10 & 0,18 & 0,00
\end{tabular}


Analisando os dados obtidos neste trabalho e os de Mendes \& Rêgo (2002), notou-se um padrão sazonal de nidificação, havendo maior número de ninhos no período de estiagem, assemelhando-se aos dados de Morato et al. (1999) na Amazônia Central, e Aguiar \& Martins (2002) em Mamanguape (PB), o que corresponde ao padrão de nidificação da maioria dos Centridini amostrados em diferentes ambientes (Heithaus 1979; Frankie et al. 1983).

No entanto, no semi-árido paraibano (Gonçalves \& Zanella 2003), Centris tarsata mostrou-se independente das estações, apresentando três picos de atividade com intervalos mais ou menos regulares de três meses: julho e novembro/ 2001 (início e meio da estação seca), fevereiro e outubro/ 2002 (meio da estação chuvosa e final da estação seca) e em janeiro/ 2003 (início das chuvas). De maio a setembro/ 2003 não houve nidificação desta espécie. Os autores concluem que uma possível ausência de diapausa induz as abelhas às atividades de fundação de ninhos durante todo o ano, principalmente na estação seca, em que há disponibilidade de recursos, tais como areia seca, óleos vegetais e pólen para as crias.

Já em um estudo realizado na Bahia (Aguiar \& Garófalo 2004), em áreas de floresta estacional semi-decídua (Baixa Grande) e de caatinga (Ipirá), C. tarsata nidificou durante o período de intensa chuva, de setembro a março em Ipirá, apresentando três gerações por ano, e de setembro a maio em Baixa Grande, onde foram observadas quatro gerações (três ciclos de vida curtos e um longo com diapausa na fase de prépupa).

A ocorrência de chuvas fortes e freqüentes, como as que ocorrem nos meses de dezembro a junho no estado do Maranhão, interrompem as atividades de nidificação das abelhas, provocando a destruição de seus ninhos, fato já relatado por Linsley (1958) para as abelhas solitárias. Gonçalves \& Zanella (2003) também comentam que a disponibilidade de chuva deve ser o fator ecológico abiótico principal a controlar os padrões de distribuição das espécies de abelhas e seus ciclos de abundância e escassez.

De acordo com Frankie et al. (1998), flutuações na frequiência de nidificação pode ser uma característica comum

Tabela II. Plantas identificadas pela análise do pólen presente em células dos ninhos de Centris tarsata em Urbano Santos, MA, Brasil.

\begin{tabular}{|c|c|c|}
\hline Famílias vegetais & $\begin{array}{l}\text { Eucaliptal } \\
\text { (EC) }\end{array}$ & $\begin{array}{c}\text { Mata Mesofítica } \\
(\mathrm{MM})\end{array}$ \\
\hline Caesalpiniaceae Kunth. & $\begin{array}{c}\text { Cassia L. } \\
\text { Hymenaea } \mathrm{L} . \\
\text { Senna } \mathrm{L} .\end{array}$ & Cassia \\
\hline Malpighiaceae Juss. & $\begin{array}{c}\text { Não identificada } \\
\text { Banisteriopsis Robinson }\end{array}$ & $\begin{array}{l}\text { Não identificada } \\
\text { Banisteriopsis } \\
\text { Byrsonima } \text { Rich. }\end{array}$ \\
\hline Myrtaceae Juss. & $\begin{array}{l}\text { Eugenia } \text { L. } \\
\text { Myrcia } \text { D. C. } \\
\text { Psidium L. } \\
\text { Não identificada }\end{array}$ & \\
\hline $\begin{array}{l}\text { Polygalaceae Juss. } \\
\text { Turneraceae D. C. }\end{array}$ & Polygala L. & $\begin{array}{l}\text { Polygala } \\
\text { Turnera L. }\end{array}$ \\
\hline
\end{tabular}
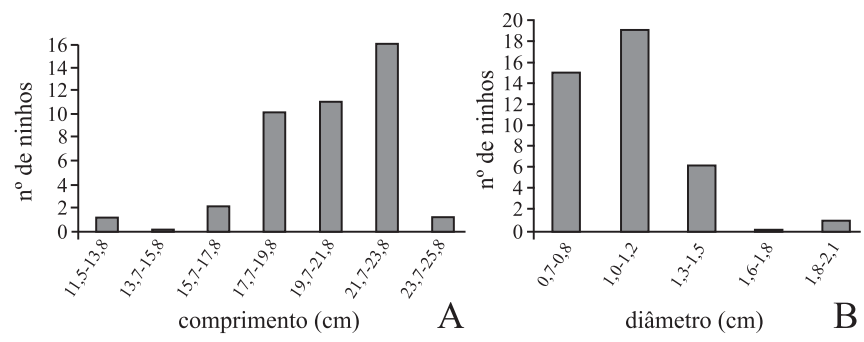

Fig. 2. (A) Comprimento e (B) diâmetro dos bambus utilizados por Centris tarsata no eucaliptal (EC) e na mata mesofítica (MM) em Urbano Santos, MA, Brasil.

nas espécies de abelhas que nidificam em cavidades preexistentes, e podem ser controladas por fatores como: diferentes taxas de mortalidade, padrões extremos nas condições climáticas anuais, presença de distúrbios nos hábitats marginais ou impacto negativo das espécies de abelhas exóticas.

Como neste estudo, na Amazônia Central, em floresta de terra firme, o número de ninhos também foi significativamente maior nas alturas entre 8 e $15 \mathrm{~m}$ (Morato et al. 1999). Isso deve ocorrer devido à presença de condições microclimáticas mais propícias à atividade de nidificação no dossel (Pérez-Maluf 1993).

Em relação ao diâmetro dos ninhos, nas dunas litorâneas de Abaeté (BA), Silva et al. (2001) verificaram 16 ninhos de Centris tarsata em madeira, sendo $31,3 \%$ com $0,8 \mathrm{~cm}$ e $68,8 \%$ com 1,0 cm de diâmetro. Em Mamanguape (PB), em área de Mata Atlântica (Aguiar \& Martins 2002), dos 63 ninhos obtidos de C. tarsata, a maioria $(53,9 \%)$ possuía $0,8 \mathrm{~cm}$ de diâmetro. Pérez-Maluf (1993) obteve seis ninhos dessa espécie em ninhos-armadilha de 0,7 a 1,2 cm de diâmetro, em Viçosa (MG). Aguiar \& Garófalo (2004) observaram ninhos de C. tarsata em bambus com 0,8 a $1,3 \mathrm{~cm}$ de diâmetro $(\mathrm{n}=28)$, sendo que a maioria possuía $0,9 \mathrm{~cm}(\mathrm{n}=11)$ ou $1,0 \mathrm{~cm}(\mathrm{n}=9)$. Provavelmente esta variação de diâmetro é favorável ao tamanho do corpo das abelhas e à construção de suas células, visto que orifícios maiores despenderiam maior gasto energético na acomodação da célula e no preenchimento dos espaços vazios (Aguiar \& Martins 2002). Isto pôde ser observado neste estudo, visto que nos bambus com orifícios maiores $(1,6-1,9 \mathrm{~cm})$ ocorreu apenas uma nidificação (Fig. 2).

Jesus \& Garófalo (2000) sugeriram que a arquitetura dos ninhos e a disposição das células dependem do espaço disponível na cavidade. No presente trabalho a variação no diâmetro e comprimento dos ninhos-armadilha não influenciou no número, disposição e medidas das células.

Segundo alguns autores (Pérez-Maluf 1993; Morato et al. 1999), a razão sexual é freqüentemente variável e associada à abundância de recursos disponíveis no ambiente para as fêmeas. Assim, as fêmeas que requerem maior quantidade de alimento para se desenvolverem, seriam preferencialmente produzidas na época em que esses recursos estariam mais abundantes. 

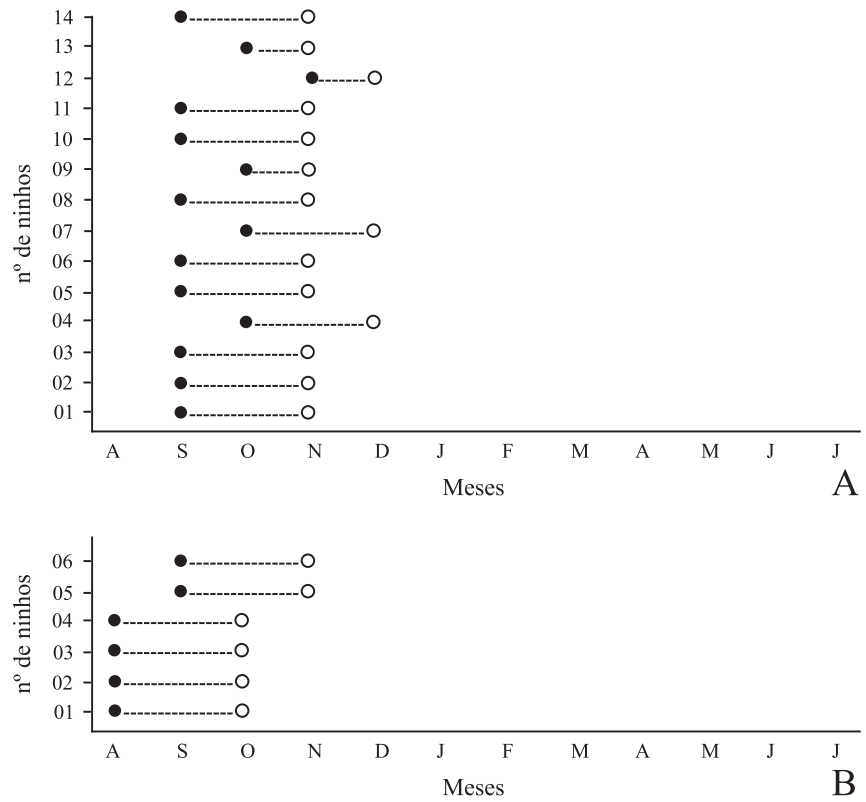

Fig. 3. Intervalo (em dias) (---) entre a coleta $(\mathbf{)})$ do ninho e a emergência (O) de indivíduos produzidos por ninhos de Centris tarsata estabelecidos no (A) eucaliptal e na (B) mata mesofítica, Urbano Santos, MA, Brasil.

Aguiar \& Martins (2002) discutiram a ação de parasitas sobre a razão sexual, inferindo que uma proporção desequilibrada entre machos e fêmeas pode ocorrer em função do parasitismo. Considerando que as células mais próximas da entrada do ninho são de machos, e que a ação dos parasitas concentra-se nestes, seria esperada uma maior emergência de fêmeas onde houvesse maior parasitismo, o que contraria os resultados apresentados. As razões sexuais diagnosticadas neste trabalho, abundância de fêmeas no EC e proporção eqüitativa entre machos e fêmeas na MM, podem estar relacionadas a outros fatores. No primeiro caso, a uma maior disponibilidade de recursos na área adjacente ao EC, e no segundo caso, ao isolamento do fragmento da MM, o qual é circundado por eucaliptos.

A disposição dos sexos nos ninhos de Centris tarsata, com as fêmeas sendo produzidas nas primeiras células construídas e os machos nas últimas células, segue o padrão observado em outras espécies de abelhas solitárias (Jesus \& Garófalo 2000; Pérez-Maluf 1993).

Em Abaeté (BA), o comprimento das células de fêmeas e machos de Centris tarsata também não apresentou diferença significativa, embora o porte corporal das fêmeas tenha sido maior, não havendo sobreposição de tamanho (Silva et al. 2001). O menor tamanho do macho pode ser resultado do consumo de alimento na fase larval, o qual é menor devido ao tamanho reduzido de suas células em relação às células da fêmea (Kim 1992).

Pérez-Maluf (1993) ressalta que a presença de células vazias entre células aprovisionadas pode reduzir a taxa de mortalidade por parasitas e patógenos, fato este observado nos ninhos do EC, os quais apresentaram maior número de células vazias e menor taxa de parasitismo. De acordo com Tepedino et al. (1979), a presença de células vazias entre células seriadas estaria associada à redução do ataque de parasitas, uma vez que encontros sucessivos de células vazias desestimulariam a procura de outras células no mesmo ninho.

A maior percentagem de parasitismo diagnosticada na mata mesofítica pode ser atribuída a dois fatores: (1) idade deste fragmento (Morato 2000), o qual é mais antigo e possui maior heterogeneidade de hábitats que o EC, que se constitui de um reflorestamento recente e homogêneo; (2) distância dos ninhos do EC em relação às áreas com maior riqueza de inimigos naturais (cerrado) (Morato 2000), ao contrário da MM, que possui diversos inimigos no seu interior, aumentando a ocorrência de parasitismo.

As espécies de Malpighiaceae, Caesalpiniaceae e Fabaceae são as principais fontes de pólen exploradas por espécies de Centris em diferentes biomas já estudados (Aguiar et al. 2003).

Banisteriopsis (Malpighiaceae) é comumente visitada por espécies de Centris (Gottsberger 1986; Sazima \& Sazima 1989; Rebêlo 1995; Albuquerque \& Mendonça 1996; Gaglianone 2003), possuindo como principal recurso o óleo, o qual é muito utilizado pelos Centridini na alimentação de suas larvas. Do mesmo modo, Byrsonima (Malpighiaceae) é bastante visitada por várias espécies de Centris para coleta de óleo e pólen (Albuquerque \& Rêgo 1989; Albuquerque \& Mendonça 1996; Aguiar et al. 2003; Westerkamp 2004; Rêgo \& Albuquerque 2006).

Cassia (Caesalpiniaceae) é uma planta com flores exclusivamente produtoras de pólen, sendo amplamente distribuídas no cerrado, florescendo principalmente nos meses de setembro e outubro (Albuquerque \& Mendonça 1996), o que corresponde aos meses de maior número de ninhos fundados neste estudo. Estas plantas são freqüentemente visitadas por espécies de Centris: C. aenea Lepeletier, 1841, C. leprieuri Spinola, 1841, C. fuscata Lepeletier, 1841, C. flavifrons Fabricius, 1775, C. tarsata e C. trigonoides (Aguiar et al. 2003).

Em Itatim (BA), Senna Mill. (Caesalpiniaceae) foi considerada uma importante fonte de pólen para Centris (Aguiar 2003), porém neste estudo foram observados poucos grãos de pólen desta planta, apenas no EC.

\section{CONCLUSÕES}

O padrão sazonal de Centris tarsata nesta região parece estar associado à floração das espécies fornecedoras de óleos e à pluviosidade. Avaliações sobre a flutuação da disponibilidade de recursos florais em cada hábitat nos períodos de estiagem e chuvoso podem auxiliar na determinação dos padrões temporais de nidificação das espécies de abelhas.

A maior abundância de ninhos no EC pode ter ocorrido devido aos seguintes fatores: ausência de diversidade de substratos naturais de nidificação na área, tornando os ninhosarmadilha fontes atrativas para nidificação e a influência da 
vegetação de entorno, que consiste em uma fonte rica em recursos naturais.

A baixa freqüência de nidificações de Centris tarsata na MM pode ter ocorrido em virtude da diversidade de substratos naturais, da umidade no interior da área e do isolamento da MM por uma cultura de eucaliptos.

A ausência de ninhos de Centridini em bambus na MC provavelmente ocorreu devido à presença de ninhos-armadilha de madeira, dispostos na mesma área logo após a colocação dos bambus, o que parece indicar uma preferência por tipos de substrato para nidificação por parte das espécies de Centris, visto que estas nidificaram em NA de bambu no ano anterior à execução deste trabalho.

O maior número de fundações diagnosticadas no estrato superior pode ter ocorrido devido ao menor grau de impacto e a condições microclimáticas mais favoráveis à nidificação no dossel.

É importante que mais trabalhos sejam realizados simultaneamente em ecossistemas com diferentes graus de preservação a fim de se obter mais informações ecológicas e dados complementares sobre a biologia de nidificação dessas abelhas.

Agradecimentos. Às instituições PIBIC / CNPq e Comercial e Agrícola Paineiras, pelo apoio financeiro e técnico-logístico, respectivamente, ao professor Dr. Carlos Alberto Garófalo, da Faculdade de Filosofia Ciências e Letras de Ribeirão Preto - USP, pelas sugestões, e à Dra ${ }^{\mathrm{a}}$. Lea Carreira, do Museu Paraense Emílio Goeldi - Belém (PA), pela identificação dos grãos de pólen.

\section{REFERÊNCIAS}

Aguiar, A. J. C.; C. A. Garófalo \& G. F. Almeida. 2005. Trap-nesting bees (Hymenoptera, Apoidea) in areas of dry semideciduous forest and caatinga, Bahia, Brazil. Revista Brasileira de Zoologia 22: 1030-1038.

Aguiar, A. J. C. \& C. F. Martins. 2002. Abelhas e vespas solitárias em ninhos-armadilha na Reserva Biológica Guaribas (Mamanguape, Paraíba, Brasil). Revista Brasileira de Zoologia 19: 101-116.

Aguiar, C. L. M. 2003. Flower visits of Centris bees (Hymenoptera: Apidae) in an area of caatinga (Bahia, Brazil). Studies on Neotropical Fauna and Environment 38: 41-45.

Aguiar, C. L. M. \& C. A. Garófalo. 2004. Nesting biology of Centris (Hemisiella) tarsata Smith (Hymenoptera, Apidae, Centridini). Revista Brasileira de Zoologia 21: 477-486.

Aguiar, C. L. M.; F. C. V. Zanella; C. F. Martins \& C. A. L. Carvalho. 2003. Plantas visitadas por Centris spp. (Hymenoptera: Apidae) na caatinga para obtenção de recursos florais. Neotropical Entomology 32: 247-259.

Albuquerque, P. M. C. \& J. A. C. Mendonça. 1996. Anthophoridae (Hymenoptera: Apoidea) e flora associada em uma formação de Cerrado no Município de Barreirinhas, MA, Brasil. Acta Amazonica 26: $45-54$

Albuquerque, P. M. C. \& M. M. C. Rêgo. 1989. Fenologia de abelhas visitantes de murici (Byrsonima crassifolia, Malpighiaceae). Boletim do Museu Paraense Emílio Goeldi 5: 163-178.

Barros, M. A. G. 1992. Fenologia da floração, estratégias reprodutivas e polinização de espécies simpátricas do gênero Byrsonima Rich (Malpighiaceae). Revista Brasileira de Biologia 52: 343-353.

Beyer, W. N.; G. W. Miller \& W. J. Fleming. 1987. Populations of trapnesting wasps and near major source of fluoride emissions in Western Tenessee. Proceedings of the Entomological Society of
Washington 89: 478-482.

Bosh, J. \& W. P. Kemp 2002. Developing and establishing bee species as crop pollination: the example of Osmia spp. (Hymenoptera: Megachilidae) and fruit trees. Bulletin of Entomologial Research 92: 3-16.

Brasil, 1984. Instituto Brasileiro de Meio Ambiente e dos Recursos Naturais Renováveis (IBAMA). Secretaria do Estado do Meio Ambiente e Turismo do Maranhão (SEMATUR). Diagnóstico dos principais problemas ambientais do Estado do Maranhão. São Luís, Lithograf. 194 p.

Buchmann, S. L. 1987. The ecology of oil flowers and their bees. Annual Review of Ecology of Ecology \& Systematics 18: 343369.

Buschini, M. L. T. 2006. Species diversity and community structure in trap-nesting bees in Southern Brazil. Apidologie 37: 58-66.

Buschini, M. L. \& L. L. Wolff. 2006. Nesting biology of Centris (Hemisiella) tarsata Smith in southern Brazil (Hymenoptera, Apidae, Centridini). Brazilian Journal of Biology 66: 1091-1101.

Camillo, E.; C. A. Garófalo; J. C. Serrano \& G. Muchillo. 1995. Diversidade e abundância sazonal de abelhas e vespas solitárias em ninhos-armadilha (Hymenoptera, Apocrita, Aculeata). Revista Brasileira de Entomologia 39: 459-470.

Coville, R. E.; G. W. Frankie \& S. B. Vinson. 1983. Nests of Centris segregata (Hymenoptera: Anthophoridae) with a review of the nesting habitats of the generous. Journal of the Kansas genus Entomolological Society 56: 109-122.

Erdtman, G. 1966. Pollen morphology and plant taxonomic. Stockholm: Almqvist \& Wilsell. 553 p.

Frankie, G. W.; S. B. Vinson \& P. A. Opler. 1983. Centris aethyctera and Centris fuscata in the lowland dry forest of Costa Rica (Hymenoptera: Anthophoridae), p. 707-708. In: D. H. Janzen (ed.). Costa Rica Natural History. Chicago, University Press. $816 \mathrm{p}$.

Frankie, G. W.; R. W. Thorp; M. Newstrom-Loyd; M. A. Rizzard; J. F. Barthel; T. L. Griswold; K. Jong-Yoon \& S. Kappagoda. 1998. Monitoring solitary bees in modified wildland habitats: implications for bee ecology and conservation. Environmental Entomology 27: $1137-1148$.

Gaglianone, M. C. 2003. Abelhas da tribo Centridini na Estação Ecológica de Jataí (Luis Antônio, SP): composição de espécies e interações com flores de Malpighiaceae, p. 279-284. In: G. A. R. Melo \& I. Alves-dos-Santos (eds.). Apoidea Neotropica: Homenagem aos 90 anos de Jesus Santiago Moure. Criciúma, Editora UNESC. 320 p.

Garófalo, C. A.; C. F Martins \& I. Alves-dos-Santos. 2004. The Brazilian solitary bee species caught in trap nests, p. 77-84. In: B. M. Freitas \& J. O. P. Pereira (eds.). Solitary bees - Conservation, rearing and management for pollination. Fortaleza, Imprensa. $285 \mathrm{p}$.

Gonçalves, A. F. \& F. C. V. Zanella. 2003. Ciclos de nidificação de abelhas e vespas solitárias que utilizam cavidades preexistentes no semi-árido paraibano. Anais do Congresso de Ecologia do Brasil 6: $322-324$.

Gottsberger, G. 1986. Some pollination strategies in neotropical savannas and florests. Plant Systematics and Evolution 152: 29-45.

Heithaus, E. R. 1979. Community structure of neotropical flower visiting bees and wasps: diversity and phenology. Ecology 60: 190202.

Jesus, B. M. V. \& C. A. Garófalo. 2000. Nesting behavior of Centris (Heterocentris) analis (Fabricius) in southeastern Brazil (Hymenoptera, Apidae, Centridini). Apidologie 31: 503-515.

Kim, J. Y. 1992. Nest dimensions of two leaf-cutter bees (Hymenoptera: Megachilidae). Annals of the Entomological Society of America 85: 85-90.

Linsley, E. G. 1958. The ecology of solitary bees. Hilgardia 27: 543599.

Loyola, R. D. \& R. P. Martins. 2006. Trap-nest occupation by solitary wasps and bees (Hymenoptera: Aculeata) in a forest urban remanent. Neotropical Entomology 35: 41-48.

Lovejoy, T. E.; R. O. Bierregaard Jr.; A. B. Rylands; J. R. Malcolm; C. 
E. Quintela; L. H. Harper; K. S. Brown Jr.; A. H. Powell; G. V. N. Powell; H. O. R. Schubart \& M. B. Hays. 1986. Edge and other effects of isolation on Amazon forest fragments, p. 257-285. In: M. E. Soule (ed.). Conservation biology: the science of scarcity and diversity. Suderland, Massachussetts Sinauer. $584 \mathrm{p}$.

Machado, I. C. 2004. Oil-collecting bees and related plants: a review of the studies in the last twenty years and case histories of plants occurring in NE Brazil, p. 255-281. In: B. M. Freitas \& J. O. P. Pereira (eds.). Solitary bees - Conservation, rearing and management for pollination. Fortaleza, Imprensa. $285 \mathrm{p}$.

Mendes, F. N. \& M. M. C. Rêgo. 2002. Nidificação de Centridini (Hymenoptera, Apidae) em ninhos-armadilha em três ecossistemas (fragmento de eucalipto, mata mesofítica e mata ciliar) no município de Urbano Santos, MA. Anais do Encontro sobre Abelhas 5: 299.

Morato, E. F. 2000. A técnica de ninhos-armadilha no estudo de comunidades de aculeata solitários. Anais do Encontro sobre Abelhas 4: 11-17.

Morato, E. F. \& L. A. O. Campos. 2000. Efeitos da fragmentação florestal sobre vespas e abelhas solitárias em uma área da Amazônia Central. Revista Brasileira de Zoologia 17: 429-444.

Morato, F. E.; M. C. Garcia \& L. A. O. Campos. 1999. Biologia de Centris Fabricius (Hymenoptera, Anthophoridae, Centridini) em matas contínuas e fragmentos na Amazônia Central. Revista Brasileira de Zoologia 16: 1213-1222.

Moreira, G. B.; L. M. Lacerda, \& M. S. Drummond. 2002. Aspectos bionômicos de Centris (Hemisiella) tarsata (Hymenoptera, Apidae) obtidos em ninhos-armadilha num fragmento de mata ciliar no Maranhão. Anais do Encontro sobre Abelhas 5: 302.

Pereira, M.; C. A. Garófalo; E. Camillo \& J. C. Serrano. 1999. Nesting biology of Centris (Hemisiella) vittata Lepeletier in southern Brazil (Hymenoptera, Apidae, Centridini). Apidologie 30: 327-338.

Perez-Maluf, R. 1993. Biologia de vespas e abelhas solitárias, em ninhos-armadilhas, em Viçosa - MG. Viçosa, MG. Universidade Federal de Viçosa. Dissertação de Mestrado. 87 p.

Rebêlo, J. M. M. 1995. Espécies de Anthophoridae (Hymenoptera, Apoidea) e suas associações com flores, numa área restrita da Ilha de São Luís, MA, Brasil. Boletim do Museu Paraense Emílio Goeldi 11: $105-124$.

Rêgo, M. M. C. \& P. M. C. Albuquerque. 1989. Comportamento das abelhas visitantes de murici, Byrsonima crassifolia (L.) Kunth, Malpighiaceae. Boletim do Museu Paraense Emílio Goeldi 5: 179-193.
Rêgo, M. M. C. \& P. M. C. Albuquerque. 2006. Polinização do Murici. São Luís, MMA/ EDUFMA. 104 p.

Roubik, D. W. 1995. Pollination of cultivated plants in the tropics. Food and Agriculture Organization of the United Nations, FAO Agricultural Services Bulletin. 118 p.

Sazima, M. \& I. Sazima. 1989. Oil-gathering bees visit flowers of eglandular morphs of the oil-producing Malpighiaceae. Botanica Acta 102: 106-111.

Sampson, B. J. \& J. H. Cane. 2000. Pollination efficiencies of three bee (Hymenoptera: Apoidea) species visiting rabbiteye blueberry. Journal of Economic Entomology 93: 1726-1731.

Silva, F. O.; B. F. Viana \& E. L. Neves. 2001. Biologia e arquitetura de ninhos de Centris (Hemisiella) tarsata Smith (Hymenoptera: Apidae: Centridini). Neotropical Entomology 30: 541-545.

Teixeira, L. A. G. \& I. C. Machado. 2000. Sistema de polinização e reprodução de Byrsonima sericea DC (Malpighiaceae). Acta Botanica Brasilica 15: 1-12.

Tepedino, V. J.; L. L. McDonald \& R. Rothwell. 1979. Defense against parasitization in mud-nesting Hymenoptera: can empty cells increase nest reproductive output? Behavioral, Ecology \& Sociobiology 6: 99-104.

Tscharntke, T.; A. Gathmann \& I. Steffandewenter. 1998. Bioindication using trap-nesting bees and wasps and their natural enemies: community structure and interactions. Journal of Applied Ecology 35: 708-719.

Viana, B. F.; F. O. Silva \& A. M. P. Kleinert. 2001. Diversidade sazonalidade de abelhas solitárias (Hymenoptera: Apoidea) em dunas litorâneas no nordeste do Brasil. Neotropical Entomology 30 245-251.

Vinson, S. B.; G. W. Frankie \& J. Barthell 1993. Threats to the diversity of solitary bees in a neotropical dry forest in Central America, p. 53-81. In: J. LaSalle \& I. D. Gauld (eds.). Hymenoptera and Biodiversity. Wallingford, CAB Internacional. 348 p.

Vinson, S. B.; H. J. Willians; G. W. Frankie \& G. Shrum. 1997. Floral lipid chemistry of Byrsonima crassifolia (Malpighiaceae) and a use of floral lipids by Centris bees (Hymenoptera: Apidae). Biotropica 29: $76-83$.

Vogel, S. 1974. Ölblumen und ölsammelnde Bienen. Tropische und Subtropische Pflanzenwelt 7: 285-369.

Westerkamp, C. 2004. Ricochet pollination in Cassias - and how bees explain enantiostyly, p. 225-230. In: B. M. Freitas \& J. O. P. Pereira (eds.). Solitary bees - Conservation, rearing and management for pollination. Fortaleza, Imprensa. 285 p.

Recebido em 23/02/2007; aceito em 25/07/2007 